\title{
MAXIMUM PRINCIPLE FOR CERTAIN GENERALIZED TIME AND SPACE FRACTIONAL DIFFUSION EQUATIONS
}

\author{
BY
}

AHMED ALSAEDI (Nonlinear Analysis and Applied Mathematics (NAAM) Research Group, Department of Mathematics, Faculty of Science, King Abdulaziz University, P.O. Box 80203, Jeddah 21589, Saudi Arabia),

BASHIR AHMAD (Nonlinear Analysis and Applied Mathematics (NAAM) Research Group, Department of Mathematics, Faculty of Science, King Abdulaziz University, P.O. Box 80203, Jeddah 21589, Saudi Arabia),

AND

MOKHTAR KIRANE (Laboratoire de Mathématiques, Image et Applications, EA 3165, Université de La Rochelle, Pôle Sciences et Technologies, Avenue Michel Crépeau, 17000 La Rochelle, France, and Nonlinear Analysis and Applied Mathematics (NAAM) Research Group, Department of Mathematics, Faculty of Science, King Abdulaziz University, P.O. Box 80203, Jeddah 21589, Saudi Arabia)

Abstract. We present an inequality for fractional derivatives related to the Leibniz rule that not only answers positively a conjecture raised by J. I. Diaz, T. Pierantozi, and L. Vázquez but also helps us to obtain a modern proof of the maximum principle for fractional differential equations. The inequality turns out to be versatile in nature as it can be used to obtain a priori estimates for many fractional differential problems.

1. Introduction. The aim of this work is to answer affirmatively a conjecture raised by J. I. Diaz, T. Pierantozi, and L. Vázquez in their interesting paper [11. Based on a huge number of simulated examples, they conjectured that for $\alpha \in(0,1)$ and $u \in C([0, T] ; \mathbb{R}), u^{\prime} \in L^{1}((0, T) ; \mathbb{R}), D_{0+}^{\alpha}$ the following inequality involving the RiemannLiouville fractional derivative (see the definition in Section 2) holds true:

$$
2 u(t) D_{0+}^{\alpha} u(t) \geq D_{0+}^{\alpha} u^{2}(t), \quad t \in[0, T] .
$$

In [1] the authors have used the former inequality for the finite time extinction phenomenon for a nonlinear fractional evolution equation with the restriction that $u$ is monotone.

Received April 11, 2013.

2010 Mathematics Subject Classification. Primary 35R11, 35B50.

Key words and phrases. Maximum principle, time fractional differential equation, time and space fractional differential equation, fractional porous medium equation.

The third author is the corresponding author.

E-mail address: aalsaedi@kau.edu.sa

E-mail address: bashirahmad_qau@yahoo.com

E-mail address: mkirane@univ-lr.fr 
We will develop a more general inequality by relaxing the monotonicity requirement that leads to (10) in a particular situation.

Moreover, relying on the inequality obtained, we will present maximum principle results for three important time and time-space fractional diffusion equations.

The first equation considered is

$$
D_{0+}^{\alpha} u=\sum_{i, j=1}^{N} \frac{\partial}{\partial x_{i}}\left(a_{i j}(x, t) \frac{\partial u}{\partial x_{j}}\right)+\sum_{j=1}^{N} b_{j}(x, t) \frac{\partial u}{\partial x_{j}}+C(x, t) u+f(x, t),
$$

which in its simplest form

$$
D_{0+}^{\alpha} u=\nabla(a(x, t) \nabla u)+f(x, t)
$$

has been considered, for example, by Benson et al. [3] to describe the dispersion through fractured rock.

The second equation we consider is the fractional porous medium equation

$$
D_{0+}^{\alpha} u=\triangle\left(|u|^{m-1} u\right),
$$

which is a generalization of the equation considered by Bologna et al. [5] (cf. also the references in [5]) which describes anomalous diffusion of Lévy-like and correlated-like in porous media, and transport of fluid in porous media and in viscous fingering [14.

The third equation is

$$
D_{0+}^{\alpha} u+(-\triangle)^{\beta} u=0,
$$

which has been considered by del-Castillo-Negrete et al. 8] and Bakunin [2] to describe the transport in magnetically confined plasmas (examples include confinement time scaling L-mode plasmas, fast propagation and nonlocal transport phenomena, nonGaussianity and long-range correlations of fluctuations, anomalous diffusion and nonGaussian pdfs in tracer transport studies).

Let us mention that in the references, one may find fractional differential equations close to those we consider in applied sciences.

\section{Preliminaries.}

Notation. Let $\Omega$ be a bounded open domain in $\mathbb{R}^{N}, N \geq 1$ with measure $d x$. $\partial \Omega$ is the boundary of $\Omega$, which is a smooth $(N-1)$-dimensional manifold, with measure $d \hat{x}$. We define by $Q=\Omega \times(0, T] .|\Omega|$ is the standard Lebesgue measure of the domain $\Omega$.

Function spaces. We will consider the following real Banach spaces of measurable functions with the norms

$$
\begin{gathered}
L^{p}(\Omega)=\left\{u(x) /\|u\|_{p}=\left(\int_{\Omega}|u(x)|^{p} d x\right)^{1 / p}<\infty\right\} ; \\
L^{\infty}(\Omega)=\left\{u(x) /\|u\|_{\infty, \Omega}=\operatorname{ess} \max _{x \in \Omega}|u(x)|<\infty\right\} ; \\
L^{\infty}(Q)=\left\{u(x, t) /\|u\|_{\infty, Q}=\operatorname{ess} \max _{(x, t) \in Q}|u(x, t)|<\infty\right\} ; \\
C([0, T])=\{u:[0, T] \rightarrow \mathbb{R} / u \text { continuous }\} ;
\end{gathered}
$$




$$
\begin{aligned}
C^{\sigma}([0, T])=\{u:[0, T] \rightarrow \mathbb{R} /|u(t)-u(t-h)|= & O\left(h^{\sigma}\right) \\
& \text { uniformly for } 0 \leq t-h<t \leq T\} .
\end{aligned}
$$

Inequalities. We shall need the following inequalities.

Young's inequality:

$$
a b \leq \frac{\varepsilon^{p}}{p} a^{p}+\frac{b^{q}}{\varepsilon^{q} q}, p+q=p q, \varepsilon>0,
$$

where $a, b$ are positive real numbers.

Hölder's inequality:

$$
\int_{\Omega}|f||g| d x \leq\left(\int_{\Omega}|f|^{p} d x\right)^{1 / p}\left(\int_{\Omega}|g|^{q} d x\right)^{1 / q}, p+q=p q, p>1, q>1 .
$$

For an integrable function $f: \mathbb{R}^{+} \rightarrow \mathbb{R}$, the Riemann-Liouville fractional integral of order $0<\alpha<1$ is defined by

$$
J_{0+}^{\alpha} f(t)=\frac{1}{\Gamma(\alpha)} \int_{0}^{t}(t-\tau)^{\alpha-1} f(\tau) d \tau, t>0,
$$

where $\Gamma(\alpha)$ is the Euler Gamma function.

For an integrable function $f: \mathbb{R}^{+} \rightarrow \mathbb{R}$, the Riemann-Liouville fractional derivative of order $0<\alpha<1$ is defined by

$$
D_{0+}^{\alpha} f(t)=\frac{1}{\Gamma(1-\alpha)} \frac{d}{d t} \int_{0}^{t}(t-\tau)^{-\alpha} f(\tau) d \tau, t>0 .
$$

For an absolutely continuous (derivable) function $f: \mathbb{R}^{+} \rightarrow \mathbb{R}$, the Caputo fractional derivative of order $0<\alpha<1$ is defined by

$$
{ }^{c} D_{0+}^{\alpha} f(t)=\frac{1}{\Gamma(1-\alpha)} \int_{0}^{t}(t-\tau)^{-\alpha} f^{\prime}(\tau) d \tau, t>0 .
$$

The relation between the Riemann-Liouville and the Caputo fractional derivatives for an absolutely continuous (derivable) function $f: \mathbb{R}^{+} \rightarrow \mathbb{R}$ is

$$
{ }^{c} D_{0+}^{\alpha} f(t)=D_{0+}^{\alpha}(f(t)-f(0)), 0<\alpha<1 .
$$

The Mittag-Leffler function of two parameters, defined by

$$
E_{\xi, \beta}(z)=\sum_{k=0}^{+\infty} \frac{z^{k}}{\Gamma(\xi k+\beta)} \quad(z, \beta \in \mathbb{C}, \operatorname{Re}(\xi)>0)
$$

admits the following properties (see Prabhakar [26, page 9):

$$
E_{\alpha, \alpha}\left(\mu t^{\alpha}\right) \leq M, t \in[a, b], a \geq 0, \mu>0,0<\alpha \leq 1,
$$

and

$$
\int_{0}^{t}(t-\tau)^{\alpha-1} E_{\alpha, \alpha}\left(\mu \tau^{\alpha}\right) d \tau<\infty
$$




\section{Main results.}

LEMMA 1. Let one of the following conditions be satisfied:

- $u \in C([0, T])$ and $v \in C^{\beta}([0, T]), \alpha<\beta \leq 1$;

- $v \in C([0, T])$ and $u \in C^{\beta}([0, T]), \alpha<\beta \leq 1$;

- $u \in C^{\beta}([0, T]), v \in C^{\delta}([0, T]), \alpha<\beta+\delta, 0<\beta<1,0<\delta<1$.

Then we have

$$
\begin{aligned}
D_{0+}^{\alpha}(u v)(t)= & u(t) D_{0+}^{\alpha} v(t)+v(t) D_{0+}^{\alpha} u(t) \\
& -\frac{\alpha}{\Gamma(1-\alpha)} \int_{0}^{t} \frac{(u(s)-u(t))(v(s)-v(t))}{(t-s)^{\alpha+1}} d s-\frac{u(t) v(t)}{\Gamma(1-\alpha) t^{\alpha}}
\end{aligned}
$$

pointwise.

Proof. Observe that

$$
u(s) v(s)=(u(s)-u(t))(v(s)-v(t))+u(t) v(s)+u(s) v(t)-u(t) v(t) .
$$

Now, by definition

$$
D_{0+}^{\alpha}(u v)(t)=\frac{1}{\Gamma(1-\alpha)} \lim _{\varepsilon \rightarrow 0} \frac{1}{\varepsilon}\left[\int_{0}^{t+\varepsilon} \frac{u(s) v(s)}{(t+\varepsilon-s)^{\alpha}} d s-\int_{0}^{t} \frac{u(s) v(s)}{(t-s)^{\alpha}} d s\right],
$$

which, using (12), can be written in the form

$$
\begin{aligned}
D_{0+}^{\alpha}(u v)(t)= & \frac{1}{\Gamma(1-\alpha)} \lim _{\varepsilon \rightarrow 0} \frac{1}{\varepsilon}\left[\int_{0}^{t+\varepsilon} \frac{(u(s)-u(t))(v(s)-v(t))}{(t+\varepsilon-s)^{\alpha}} d s\right. \\
& -\int_{0}^{t} \frac{(u(s)-u(t))(v(s)-v(t))}{(t-s)^{\alpha}} d s \\
& +u(t)\left\{\int_{0}^{t+\varepsilon} \frac{v(s)}{(t+\varepsilon-s)^{\alpha}} d s-\int_{0}^{t} \frac{v(s)}{(t-s)^{\alpha}} d s\right\} \\
& +v(t)\left\{\int_{0}^{t+\varepsilon} \frac{u(s)}{(t+\varepsilon-s)^{\alpha}} d s-\int_{0}^{t} \frac{u(s)}{(t-s)^{\alpha}} d s\right\} \\
& \left.-u(t) v(t)\left\{\int_{0}^{t+\varepsilon} \frac{1}{(t+\varepsilon-s)^{\alpha}} d s-\int_{0}^{t} \frac{1}{(t-s)^{\alpha}} d s\right\}\right] \\
=u(t) D_{0+}^{\alpha} v(t)+v(t) D_{0+}^{\alpha} u(t)-\frac{u(t) v(t)}{\Gamma(1-\alpha) t^{\alpha}} & \\
& -\frac{\alpha}{\Gamma(1-\alpha)} \int_{0}^{t} \frac{(u(s)-u(t))(v(s)-v(t))}{(t-s)^{\alpha+1}} d s .
\end{aligned}
$$

Consequences. The immediate consequences are listed below.

1. In the case where $u$ and $v$ have the same sign and are both increasing or both decreasing, then

$$
D_{0+}^{\alpha}(u v)(t) \leq u(t) D_{0+}^{\alpha} v(t)+v(t) D_{0+}^{\alpha} u(t) .
$$

By setting $u=v$ in inequality (15) and requiring only $u \in C^{\beta}([0, T]), \alpha<2 \beta, \beta \leq 1$, we obtain the inequality conjectured by J. I. Diaz, T. Pierantozi, and L. Vázquez:

$$
2 u(t) D_{0+}^{\alpha} u(t) \geq D_{0+}^{\alpha} u^{2}(t) .
$$


In the case $\beta<1$, our requirement on $u$ is weaker than the one of [1] as $u$ is not differentiable. However, in the case $\beta=1$, by Rademacher's theorem $u$ is almost everywhere differentiable [12].

2. By induction, one can show that, for any integer $p \geq 2$,

$$
p u^{(p-1)}(t) D_{0+}^{\alpha} u(t) \geq D_{0+}^{\alpha} u^{p}(t) \quad\left\{\begin{array}{l}
\text { for } p \\
\text { for } \quad p \quad \text { oden, }
\end{array}\right.
$$

It is inequality (17) for $p$ even that we will use hereafter.

Remark 1. For the Caputo derivative, inequality (15) reads

$$
\begin{aligned}
{ }^{c} D_{0+}^{\alpha}(u v)(t) \leq u(t)^{c} D_{0+}^{\alpha} v(t)+v(t)^{c} D_{0+}^{\alpha} u(t) & \\
& +\frac{t^{-\alpha}}{\Gamma(1-\alpha)}(u(t) v(0)+v(t) u(0)-u(0) v(0)) .
\end{aligned}
$$

\section{Applications.}

1. The main result in [1] remains true without requiring the solution to be monotone increasing.

2. We create a modern proof of the maximum principle for the fractional diffusion equation.

Maximum principle for partial fractional differential equations. The maximum principle for fractional-in-time diffusion equations has been dealt with in a series of very interesting papers by Luchko [21], 22, and [23]. Our lines of proofs for the different problems under consideration differ from the one used by Luchko, for example, in [23].

1. Iteration proof of the maximum principle for the fractional heat equation.

Here, we use the iteration technique to prove a version of the maximum principle for the fractional heat problem in divergence form. We consider

$$
D_{0+}^{\alpha} u=\sum_{i, j=1}^{N} \frac{\partial}{\partial x_{i}}\left(a_{i j}(x, t) \frac{\partial u}{\partial x_{j}}\right)+\sum_{j=1}^{N} b_{j}(x, t) \frac{\partial u}{\partial x_{j}}+C(x, t) u+f(x, t)
$$

in $Q=\Omega \times(0, T]$, where $\Omega \subset \mathbb{R}^{N}$ is a bounded domain with regular boundary $\partial \Omega$, supplemented either with the von Neumann boundary condition

$$
\partial u / \partial \eta=0, x \in \partial \Omega, t>0,
$$

where $\eta$ is the outward normal, or the Dirichlet boundary condition

$$
u=0, x \in \partial \Omega, t>0,
$$

and the initial condition

$$
\lim _{t \rightarrow 0} \Gamma(\alpha) t^{1-\alpha} u(t, x)=\lim _{t \rightarrow 0}\left(J_{0+}^{1-\alpha} u\right)(x, t)=u_{0}(x), x \in \Omega .
$$

Assume that

$\left(H_{1}\right)$ the function $u$ satisfying (18) is in $\mathcal{C}(\bar{Q})$ and has derivatives $\partial u / \partial x_{i}, \partial^{2} u / \partial x_{i} \partial x_{j}$ in $\mathcal{C}(Q), \frac{t^{-\alpha}}{\Gamma(1-\alpha)} * u \in C^{1}((0, T] ; C(\Omega))$; 
$\left(H_{2}\right) a_{i j}, \partial / \partial x_{i}\left(a_{i j}\right)$ are in $\mathcal{C}(\bar{Q})$ and for every $(x, t) \in Q$ and every $\xi \in \mathbb{R}^{N}$

$$
\sum_{i, j=1}^{N} a_{i j}(x, t)\left(\xi_{i}-\xi_{j}\right) \geq 0 ;
$$

$\left(H_{3}\right)$ for a positive constant $d, C(x, t) \leq-d$ in $Q$;

$\left(H_{4}\right) f$ is uniformly bounded, $|f(x, t)| \leq M$ in $Q$.

The aim is to obtain a bound on $\|u(., t)\|_{L^{\infty}(\Omega)}$ in terms of $\|u(., 0)\|_{L^{\infty}}, \mathcal{N}(t)=$ $\max _{t \geq 0}\|f(., t)\|_{L^{\infty}(\Omega)}$ and $m=\max _{(x, t)}|u(x, s)|$ for $(x, t) \in \partial \Omega \times(0, T]$.

Theorem 1. If $b_{j},\left(b_{j}\right)_{x_{j}}$ are continuous in $Q$ for $j=1, \ldots, N$ and $u$ satisfies (18), then for every $0<t \leq T$, it holds that

$$
\|u(t, .)\|_{\infty, \Omega} \leq T\|f(., .)\|_{\infty, Q}+C_{0}\|u(., 0)\|_{\infty, \Omega}+\frac{3}{2}\|u(., .)\|_{\infty, \partial \Omega \times(0, T]},
$$

where $C_{0}$ is a positive constant.

Proof. We proceed by multiplying each member of (18) by $u^{2^{k}-1}(k \in \mathbb{N}, k \gg 1)$ throughout and then integrate over $\Omega$. Thus,

$$
\begin{aligned}
\int_{\Omega}\left(D_{0+}^{\alpha} u\right) u^{2^{k}-1} d x & =\int_{\Omega} \sum_{i, j=1}^{N} \frac{\partial}{\partial x_{i}}\left(a_{i j}(x, t) \frac{\partial u}{\partial x_{j}}\right) u^{2^{k}-1} d x \\
& +\int_{\Omega} \sum_{j=1}^{N} b_{j}(x, t) \frac{\partial u}{\partial x_{j}} u^{2^{k}-1} d x+\int_{\Omega} C(x, t) u^{2^{k}} d x \\
& +\int_{\Omega} f(x, t) u^{2^{k}-1} d x .
\end{aligned}
$$

After integrating by parts, using inequality (17) and the Hölder inequality with $q=$ $2^{k}, p=2^{k}\left(2^{k}-1\right)^{-1},(p+q=p q)$ for the fourth term, we obtain

$$
\begin{aligned}
2^{-k} D_{0+}^{\alpha} \int_{\Omega} u^{2^{k}} d x & \leq \int_{\partial \Omega} \sum_{i, j=1}^{N} a_{i j} \frac{\partial u}{\partial x_{j}} \cos \left(\eta, x_{j}\right) u^{2^{k}-1} d \hat{x} \\
& -\left(2^{k}-1\right) \int_{\Omega} \sum_{i, j=1}^{N} a_{i j} \frac{\partial u}{\partial x_{i}} \frac{\partial u}{\partial x_{j}} u^{2^{k}-2} d x \\
& +2^{-k} \int_{\partial \Omega} \sum_{j=1}^{N} b_{j} \cos \left(\eta, x_{j}\right) u^{2^{k}} d \hat{x}-2^{-k} \int_{\Omega} \sum_{j=1}^{N} \frac{\partial b_{j}}{\partial x_{j}} u^{2^{k}} d x \\
& +\int_{\Omega} C(x, t) u^{2^{k}} d x+\left(\int_{\Omega}|f|^{2^{k}} d x\right)^{2^{-k}}\left(\int_{\Omega} u^{2^{k}} d x\right)^{1-2^{-k}} .
\end{aligned}
$$

Using $\left(H_{2}\right),\left(H_{3}\right)$ and the fact that $a_{i j}, b_{j}, u, \partial a_{i j} / \partial x_{i}, \partial b_{j} / \partial x_{j}, \partial u / \partial x_{i}, \partial^{2} u / \partial x_{i} \partial x_{j}$ are continuous in $\bar{Q}$, we obtain

$$
\begin{aligned}
D_{0+}^{\alpha} \int_{\Omega} u^{2^{k}} d x & \leq 2^{k} m^{2^{k}-1} C_{1}+m^{2^{k}} C_{2}+\int_{\Omega} C_{3}(x, t) u^{2^{k}} d x \\
& +2^{k} \mathcal{N}(t)|\Omega|^{2^{-k}}\left(\int_{\Omega} u^{2^{k}} d x\right)^{1-2^{-k}},
\end{aligned}
$$


where $C_{1}$ and $C_{2}$ are positive constants such that

$$
\begin{aligned}
& C_{1} \geq \max _{t_{0} \leq t \leq T} \int_{\partial \Omega}\left|\sum_{i, j=1}^{N} a_{i j} \frac{\partial u}{\partial x_{j}} \cos \left(\eta, x_{j}\right)\right| d \hat{x}, \\
& C_{2} \geq \max _{t_{0} \leq t \leq T} \int_{\partial \Omega} \sum_{j=1}^{N} b_{j} \cos \left(\eta, x_{j}\right) d \hat{x}, \\
& C_{3}(x, t)=2^{k} C(x, t)-\sum_{j=1}^{N} \frac{\partial b_{j}}{\partial x_{j}} .
\end{aligned}
$$

Since $\partial b_{j} / \partial x_{j}$ is bounded and $C(x, t) \leq-d<0$, then $C_{3}(x, t)$ is negative for sufficiently large $k$.

Now we set

$$
\begin{gathered}
y_{k}(t)=\|u(., t)\|_{L^{2 k}(\Omega)}^{2^{k}}, \\
b_{k}(t)=\mathcal{N}(t)|\Omega|^{2^{-k}}, \\
\beta=2^{-k}, \quad a_{k}=2 m^{2^{k}-1}\left(2^{k} C_{1}+m C_{2}\right),
\end{gathered}
$$

and fix $\tilde{a}>0$ such that $C_{3}(x, t) \leq-\tilde{a}$ for all $(x, t) \in Q$.

- If $m=0$ (equivalently $a_{k}=0$ ), then from (25) we obtain the fractional differential inequality:

$$
D_{0+}^{\alpha} y_{k}(t) \leq-\tilde{a} y_{k}(t)+\beta^{-1} b_{k}(t)\left(y_{k}(t)\right)^{1-\beta} .
$$

Next we attain a bound on $y_{k}(t)$.

Using (2) with $a=b_{k}(t), b=\left(y_{k}(t)\right)^{1-\beta}$ and $\frac{1}{1-\beta}=q>1, p=\frac{1}{\beta}$, we find that

$$
\beta^{-1} b_{k}(t)\left(y_{k}(t)\right)^{1-\beta} \leq \varepsilon^{p} b_{k}^{p}(t)+\frac{1}{\varepsilon^{q} q} y_{k}(t) \leq \varepsilon^{p} b_{k}^{p}(t)+\frac{1-\beta}{\beta \varepsilon^{\frac{1}{1-\beta}}} y_{k}(t) .
$$

Whereupon

$$
D_{0+}^{\alpha} y_{k}(t) \leq-\left(\tilde{a}-\frac{1-\beta}{\beta \varepsilon^{\frac{1}{1-\beta}}}\right) y_{k}(t)+\varepsilon^{p} b_{k}^{p}(t) .
$$

Inequality (27) can be rewritten in the form

$$
D_{0+}^{\alpha}\left(y_{k}(t)-y_{k}(0)\right) \leq \lambda_{k}\left(y_{k}(t)-y_{k}(0)\right)+\lambda_{k} y_{k}(0)+\varepsilon^{p} b_{k}^{p}(t),
$$

where we have set

$$
\lambda_{k}=-\tilde{a}+\frac{1-\beta}{\beta \varepsilon^{\frac{1}{1-\beta}}} .
$$

According to [18, p. 230], the solution of (28) can be estimated as

$$
\begin{aligned}
y_{k}(t) \leq \quad & y_{k}(0)+\lambda_{k} y_{k}(0) \int_{0}^{t}(t-s)^{\alpha-1} E_{\alpha, \alpha}\left(\lambda_{k}(t-s)^{\alpha}\right) d s \\
& +\varepsilon^{p} \int_{0}^{t}(t-s)^{\alpha-1} E_{\alpha, \alpha}\left(\lambda_{k}(t-s)^{\alpha}\right) b_{k}^{p}(s) d s .
\end{aligned}
$$


Using the inequality $\sqrt[n]{A+B} \leq \sqrt[n]{A}+\sqrt[n]{B}, A, B>0, n>1, n \in \mathbb{N}$, we can transform (29) into

$$
\begin{aligned}
y_{k}^{\beta}(t) & \leq y_{k}^{\beta}(0)+\lambda_{k}^{\beta} y_{k}^{\beta}(0)\left(\int_{0}^{t}(t-s)^{\alpha-1} E_{\alpha, \alpha}^{\beta}\left(\lambda_{k}(t-s)^{\alpha}\right) d s\right)^{\beta} \\
& +\left.\left.\varepsilon^{p \beta}\left(\int_{0}^{t}(t-s)^{\alpha-1} E_{\alpha, \alpha}\left(\lambda_{k}(t-s)^{\alpha}\right) d s\right)^{\beta}|\mathcal{N}(t)| \Omega\right|^{2-k}\right|^{p \beta} .
\end{aligned}
$$

Observe that $p \beta=1$ and $\beta \rightarrow 0, y_{k}^{\beta}(t) \rightarrow\|u(., t)\|_{L^{\infty}(\Omega)}$ as $k \rightarrow \infty$. We set $\varepsilon=t \geq \varepsilon_{0}>0$. Thus, taking the limit as $k \rightarrow+\infty$ in inequality (30), we obtain

$$
\|u(., t)\|_{\infty, \Omega} \leq T\|f(., .)\|_{\infty, Q}+C_{0}\|u(., 0)\|_{\infty, \Omega},
$$

for $0<t \leq T$ and $C_{0}=1+\varepsilon_{0}^{-1}$.

- If $m \neq 0$, proceeding as before we obtain the inequality

$$
D_{0+}^{\alpha}\left(y_{k}(t)-y_{k}(0)\right) \leq \lambda_{k}\left(y_{k}(t)-y_{k}(0)\right)+\Lambda_{k}+\varepsilon^{p} b_{k}^{p}(t),
$$

where we have set

$$
\Lambda_{k}=\lambda_{k} y_{k}(0)+a_{k}
$$

An integration of the inequality (31) leads to the estimate

$$
\|u(t, .)\|_{\infty, \Omega} \leq T\|f(., .)\|_{\infty, Q}+C_{0}\|u(., 0)\|_{\infty, \Omega}+\frac{3}{2}\|u(., .)\|_{\infty, \partial \Omega \times(0, T]}
$$

for $0<t \leq T$.

2. The maximum principle for the fractional heat equation with a nonlinear diffusion.

For this part we need the Stroock-Varopoulos inequality, which is well known in the theory of sub-Markovian operators (cf. for example [28, [30]). We recall it in the following lemma.

Lemma 2. Let $0<\beta \leq 1,1<p$. Then

$$
\int_{D}\left(|\varphi|^{p-2} \varphi\right)(-\Delta)^{\beta} \varphi \geq\left.\left.\frac{4(p-1)}{p^{2}} \int_{D}\left|(-\Delta)^{\beta / 2}\right| \varphi\right|^{p / 2}\right|^{2}
$$

for all $\varphi \in L^{p}(D)$ such that $(-\Delta)^{\beta / 2} \varphi \in L^{p}(D)$ ( $D$ a bounded domain or $\mathbb{R}^{d}$ ).

Let us consider the following porous medium equation with time fractional derivative

$$
\begin{gathered}
D_{0^{+}}^{\alpha} u=\Delta\left(|u|^{m-1} u\right), \quad 0<\alpha<1<m, x \in \Omega, t>0, \\
\lim _{t \rightarrow 0} \Gamma(\alpha) t^{1-\alpha} u(t, x)=\lim _{t \rightarrow 0}\left(J_{0+}^{1-\alpha} u\right)(x, t)=u(x, 0), x \in \Omega, \\
u(x, t)=0, x \in \partial \Omega, t>0 .
\end{gathered}
$$

Theorem 2. Let $u_{0} \geq 0$ and $u$ be the solution to problem (33), (34) and (35). Then, we have

$$
\|u(., t)\|_{\infty, \Omega} \leq\|u(., 0)\|_{\infty, \Omega}
$$


Proof. First, as $u(x, 0) \geq 0$, then $u(x, t) \geq 0$. Multiplying each term of (33) by $p u^{p-1}\left(p \in \mathbb{N}^{*}\right)$, and integrating over $\Omega$, we obtain

$$
\int_{\Omega} p u^{p-1} D_{0^{+}}^{\alpha} u d x-p \int_{\Omega} u^{p-1} \Delta u^{m} d x=0 .
$$

But

$$
\begin{aligned}
\int_{\Omega} u^{p-1} \Delta u^{m} d x & =\int_{\partial \Omega} u^{p-1} u^{m-1} \frac{\partial}{\partial \eta} u d \sigma-\int_{\Omega}(p-1) u^{p-2} u^{m-1}|\nabla u|^{2} d x \\
& =-\int_{\Omega}(p-1) u^{p-2} u^{m-1}|\nabla u|^{2} d x .
\end{aligned}
$$

If $p>1$, then

$$
p \int_{\Omega} u^{p-1} D_{0^{+}}^{\alpha} u d x+p(p-1) \int_{\Omega} u^{p-2} u^{m-1}|\nabla u|^{2} d x=0 .
$$

Now, using the inequality (17), we get

$$
\int_{\Omega} D_{0^{+}}^{\alpha} u^{p} d x+\frac{4 p(p-1)}{(p+m-1)^{2}} \int_{\Omega}\left|\nabla u^{\frac{p+m-1}{2}}\right|^{2} d x \leq 0 .
$$

As a first consequence, we obtain

$$
D_{0^{+}}^{\alpha} \int_{\Omega} u^{p} d x \leq 0 \quad \Longrightarrow \quad D_{0^{+}}^{\alpha}\left(\int_{\Omega} u^{p} d x-\int_{\Omega} u_{0}^{p} d x\right) \leq 0 .
$$

Whereupon

$$
\int_{\Omega} u^{p} d x \leq \int_{\Omega} u_{0}^{p} d x
$$

We finally conclude that

$$
\|u(., t)\|_{\infty, \Omega} \leq\|u(., 0)\|_{\infty, \Omega} .
$$

REMARK 2. In the case where $\Omega=\mathbb{R}^{N}$, we still have inequality (41). So,

$$
\|u(., t)\|_{p} \leq\|u(., 0)\|_{p}
$$

for any $1<p<\infty$. The limit cases $p=1$ and $p=\infty$ can be obtained by an elliptic approximation.

3. The maximum principle for the time and space fractional heat equation.

In 23] Luchko raised the problem of the maximum principle for time-space fractional diffusion equations. However, he did not fix the type of space fractional derivatives that have to be considered as several options were available. One may consider the regional fractional Laplacian [4] or a fractional power of the Laplacian from spectral theory or a sum of space fractional derivatives in the sense of Riemann-Liouville with respect to each direction such as $\sum_{i=1}^{i=N} \partial^{\alpha_{i}} / \partial x_{i}^{\alpha_{i}}$, where $\partial^{\alpha_{i}} / \partial x_{i}^{\alpha_{i}}$ stands for the Riemann-Liouville fractional derivative in the direction $x_{i}$.

Here we give some results in certain situations.

Let us consider the problem

$$
\left\{\begin{array}{l}
D_{0^{+}}^{\alpha} u+(-\Delta)^{\beta} u=0, \quad 0<\alpha<1,0<\beta \leq 1, x \in \Omega, t>0 \\
u=0 \text { or } \partial u / \partial \eta=0, x \in \partial \Omega, t>0 \\
\lim _{t \rightarrow 0} \Gamma(\alpha) t^{1-\alpha} u(t, x)=\lim _{t \rightarrow 0}\left(J_{0+}^{1-\alpha} u\right)(x, t)=u_{0}(x)
\end{array}\right.
$$


where $\Omega$ is a bounded domain. Here, $\Delta$ is the Laplace operator, the fractional power $(-\Delta)^{\beta}$ is a positive self-adjoint operator; it can be described by the spectral theorem. Let $\left\{\varphi_{k}, \lambda_{k}\right\}$ denote an orthonormal basis of $L^{2}(\Omega)$ consisting of eigenfunctions of $-\Delta$ in $\Omega$ with homogeneous Dirichlet boundary conditions and their corresponding eigenvalues. The operator $(-\Delta)^{\beta}$ is defined for $u \in C_{0}^{\infty}(\Omega), u=\Sigma_{k=1}^{\infty} u_{k} \varphi_{k}$, by

$$
(-\Delta)^{\beta} u=\sum_{k=1}^{\infty} \lambda_{k}^{\beta} u_{k} \varphi_{k}
$$

This operator can be extended by density for $u$ in the Hilbert space

$$
H_{0}^{\beta}(\Omega)=\left\{u \in L^{2}(\Omega):\|u\|_{H_{0}^{\beta}}^{2}=\sum_{k=1}^{\infty} \lambda_{k}^{\beta} u_{k}^{2}<\infty\right\} .
$$

By the spectral theorem, one has

$$
e^{-t(-\Delta)^{\beta}}=\int_{0}^{t} \Phi_{t, \beta}(s) e^{s \Delta} d s
$$

for $t>0$, where $\Phi_{t, \beta}$ has the property

$$
e^{-t \lambda^{\beta}}=\int_{0}^{t} \Phi_{t, \beta}(s) e^{-s \lambda} d s, \quad \lambda>0 .
$$

The fact that

implies

$$
(-1)^{k} \frac{\partial^{k}}{\partial \lambda^{k}} e^{-t \lambda^{\beta}} \geq 0, \quad \lambda, t>0, k \in \mathbb{Z}^{+}
$$

$$
\Phi_{t, \beta}(s) \geq 0, \quad 0 \leq \lambda<\infty, t>0 .
$$

As the solution of (42) is given by

$$
u(t)=E_{\alpha, \alpha}\left(-t^{\alpha}(-\Delta)^{\beta}\right) u_{0},
$$

where the linear operator $E_{\alpha, \alpha}\left(-t^{\alpha}(-\Delta)^{\beta}\right)$ is given by the standard operator calculus for self-adjoint operators. As derived in (A.37) of [24], one has

$$
E_{\alpha, \alpha}(-s)=\int_{0}^{\infty} M_{\alpha}(r) e^{-r s} d r, s>0
$$

with

$$
M_{\alpha}(r)=\frac{1}{2 \pi i} \int_{\gamma} e^{\sigma-r \sigma^{\alpha}} \frac{d \sigma}{\sigma^{1-\alpha}},
$$

where $\gamma$ can be taken as a vertical line $\{i \varrho+\varepsilon: \varrho \in \mathbb{R}\}$, with small $\varepsilon>0$. It follows that

$$
E_{\alpha, \alpha}\left(-t^{\alpha}(-\Delta)^{\beta}\right)=\int_{0}^{\infty} M_{\alpha}(r) e^{-r t^{\alpha}} d r, t>0 .
$$

As noted in [24, (A.41)],

$$
\alpha \int_{0}^{\infty} r^{-1-\alpha} M_{\alpha}\left(r^{-\alpha}\right) e^{-r s} d r=e^{-s^{\alpha}}
$$

which compared with (43) gives

$$
\alpha r^{-1-\beta} M_{\alpha}\left(r^{-\alpha}\right)=\Phi_{1, \alpha}(r) .
$$

Thus it follows that $M_{\alpha}(r) \geq 0$. 
Theorem 3. Let $u$ be the solution to (42) with $0 \leq u_{0}(x)$. Then we have

$$
\|u(., t)\|_{\infty, \Omega} \leq\left\|u_{0}\right\|_{\infty, \Omega} .
$$

Proof. As $0 \leq u_{0}(x)$, then $0 \leq u(x, t)$. Now, multiplying each term of the first equation of (42) by $p u^{p-1}$ and then integrating over $\Omega$, we obtain

$$
D_{0^{+}}^{\alpha} \int_{\Omega} u^{p} d x+\frac{4(p-1)}{p} \int_{\Omega}\left(A^{\alpha / 2}\left(\phi^{p / 2}\right)\right)^{2} d x \leq 0,
$$

thanks to the Stroock-Varopoulos inequality (Lemma 2).

Consequently, we have

$$
D_{0^{+}}^{\alpha} \int_{\Omega} u^{p} d x \leq 0
$$

As in the proof of the previous theorem, we conclude that

$$
\|u(., t)\|_{\infty, \Omega} \leq\left\|u_{0}\right\|_{\infty, \Omega} .
$$

REMARK 3. In the case where $\Omega=\mathbb{R}^{N}$, we have

$$
\|u(., t)\|_{p} \leq\|u(., 0)\|_{p}
$$

for $1<p<\infty$. However in this case, the nonlocal operator $(-\triangle)^{\beta}$ is defined as follows for any function $u$ in the Schwartz class through the Fourier transform: if $(-\triangle)^{\beta} u=v$, then

$$
\hat{v}(\xi)=|\xi|^{2 \beta} \hat{u}(\xi)
$$

where $\xi$ is the Fourier variable.

If $0<\beta<1$, the nonlocal operator $(-\triangle)^{\beta}$ is defined by its Riesz representation

$$
(-\triangle)^{\beta} u(x)=C_{N, \beta} P . V \int_{\mathbb{R}^{N}} \frac{u(x)-u(y)}{|x-y|^{d+2 \beta}},
$$

where $C_{d, \beta}$ is a normalization constant (cf. 20]).

For example, following the same lines as before, a maximum principle can be obtained for the equation

$$
D_{0^{+}}^{\alpha} u+(-\Delta)^{\beta} u^{m}=f(x, t), \quad 0<\alpha<1, x \in \Omega, t>0 .
$$

Acknowledgments. This paper was funded by the Deanship of Scientific Research (DSR), King Abdulaziz University (KAU), Jeddah Saudi Arabia, under grant No. $(15 / 34 / \mathrm{Gr})$. The authors, therefore, acknowledge technical and financial support of KAU.

We thank very much the referees for their remarks.

\section{References}

[1] Paul S. Addison, Bo Qu, Alistair Nisbet, and Gareth Pender, A non-Fickian, particle-tracking diffusion model based on fractional Brownian motion, Internat. J. Numer. Methods Fluids 25 (1997), no. 12, 1373-1384, DOI 10.1002/(SICI)1097-0363(19971230)25:12〈1373::AID-FLD620 $\rangle$ 3.3.CO;2-Y. MR 1601533 (98i:86001)

[2] Oleg G. Bakunin, Turbulence and diffusion, scaling versus equations, Springer Series in Synergetics, Springer-Verlag, Berlin, 2008. MR2450437 (2009j:76001)

[3] D. A. Benson, S. W. Wheatcraft, and M. M. Meerschaert, Application of a fractional advectiondispersion equation, Water Resources Research Vol. 36, No. 6 (2000), 1403-1412. 
[4] Krzysztof Bogdan, Krzysztof Burdzy, and Zhen-Qing Chen, Censored stable processes, Probab. Theory Related Fields 127 (2003), no. 1, 89-152, DOI 10.1007/s00440-003-0275-1. MR2006232 (2004g:60068)

[5] M. Bologna, C. Tsallis, P. Grigolini, Anomalous diffusion associated with nonlinear fractional derivative Fokker-Planck-like equation: Exact time-dependent solutions, Phys. Review E 62 (2) (2000), 2213.

[6] Jean-Philippe Bouchaud and Marc Potters, Theory of financial risks, Cambridge University Press, Cambridge, 2000. From statistical physics to risk management; With a foreword by Nick Dunbar. MR.1787145 (2001f:91001)

[7] D. Del-Castillo-Negrete, B.A. Carreras and V.E. Lynch, Fractional diffusion in plasma turbulence. Physics of Plasmas 11, (2004), 3854.

[8] D. Del-Castillo-Negrete, B.A. Carreras and V.E. Lynch, Nondiffusive transport in plasma turbulence: a fractional diffusion approach. Physical Review Letters 94, 065003 (2005).

[9] D. Del-Castillo-Negrete, Fractional diffusion models of transport in magnetically confined plasmas. Proceedings of the 32nd European Physical Society, Plasma Physics Conference. Tarragona Spain (2005).

[10] D. del-Castillo-Negrete, Fractional diffusion models of nonlocal transport, Phys. Plasmas 13 (2006), no. 8, 082308, 16, DOI 10.1063/1.2336114. MR2249732 (2007c:76081)

[11] J. I. Diaz, T. Pierantozi and L. Vázquez, On the finite time extinction phenomena for some nonlinear fractional evolution equations. Preprint.

[12] Lawrence C. Evans and Ronald F. Gariepy, Measure theory and fine properties of functions, Studies in Advanced Mathematics, CRC Press, Boca Raton, FL, 1992. MR1158660 (93f:28001)

[13] B. R. Gadjiev, Disorder and critical phenomena, arxiv.org/pdf/0809.5036, 2008.

[14] P. Grosfils, and J. B. Boon, Nonextensive statistics in viscous fingering, Phys. A 362 (1) (2006) 168.

[15] G. H. Hardy and J. E. Littlewood, Some properties of fractional integrals. I, Math. Z. 27 (1928), no. 1, 565-606, DOI 10.1007/BF01171116. MR.1544927

[16] Applications of fractional calculus in physics, World Scientific Publishing Co. Inc., River Edge, NJ, 2000. Edited by R. Hilfer. MR.1890104 (2002j:00009)

[17] Rudolf Gorenflo and Sergio Vessella, Abel integral equations, analysis and applications, Lecture Notes in Mathematics, vol. 1461, Springer-Verlag, Berlin, 1991. MR1095269 (92e:45003)

[18] Anatoly A. Kilbas, Hari M. Srivastava, and Juan J. Trujillo, Theory and applications of fractional differential equations, North-Holland Mathematics Studies, vol. 204, Elsevier Science B.V., Amsterdam, 2006. MR2218073 (2007a:34002)

[19] A. N. Kochuber,, The Cauchy problem for evolution equations of fractional order (Russian), Differentsial'nye Uravneniya 25 (1989), no. 8, 1359-1368, 1468; English transl., Differential Equations 25 (1989), no. 8, 967-974 (1990). MR1014153 (90g:34063)

[20] N. S. Landkof, Foundations of modern potential theory, Springer-Verlag, New York, 1972. Translated from the Russian by A. P. Doohovskoy; Die Grundlehren der mathematischen Wissenschaften, Band 180. MR0350027 (50 \#2520)

[21] Yury Luchko, Maximum principle for the generalized time-fractional diffusion equation, J. Math. Anal. Appl. 351 (2009), no. 1, 218-223, DOI 10.1016/j.jmaa.2008.10.018. MR2472935 (2009m:35274)

[22] Yury Luchko, Some uniqueness and existence results for the initial-boundary-value problems for the generalized time-fractional diffusion equation, Comput. Math. Appl. 59 (2010), no. 5, 1766-1772, DOI 10.1016/j.camwa.2009.08.015. MR2595950(2010k:35515)

[23] Yuri Luchko, Initial-boundary-value problems for the one-dimensional time-fractional diffusion equation, Fract. Calc. Appl. Anal. 15 (2012), no. 1, 141-160, DOI 10.2478/s13540-012-0010-7. MR:2872116

[24] F. Mainardi, P. Paradisi, and R. Gorenflo, Probabililty distributions generated by fractional diffusion equation, Preprint, 2004.

[25] Ralf Metzler and Joseph Klafter, The random walk's guide to anomalous diffusion: a fractional dynamics approach, Phys. Rep. 339 (2000), no. 1, 77, DOI 10.1016/S0370-1573(00)00070-3. MR.1809268 (2001k:82082)

[26] Tilak Raj Prabhakar, A singular integral equation with a generalized Mittag Leffler function in the kernel, Yokohama Math. J. 19 (1971), 7-15. MR.0293349 (45 \#2426) 
[27] Stefan G. Samko, Anatoly A. Kilbas, and Oleg I. Marichev, Fractional integrals and derivatives, theory and applications, Gordon and Breach Science Publishers, Yverdon, 1993. Edited and with a foreword by S. M. Nikol'skiur; Translated from the 1987 Russian original; Revised by the authors. MR 1347689 (96d:26012)

[28] D. W. Stroock, An introduction to the theory of large deviations, Universitext, Springer-Verlag, New York, 1984. MR755154(86h:60067a)

[29] Vladimir V. Uchaikin and Renat T. Sibatov, Fractional theory for transport in disordered semiconductors, Commun. Nonlinear Sci. Numer. Simul. 13 (2008), no. 4, 715-727, DOI 10.1016/j.cnsns.2006.07.008. MR.2381497(2008k:82136)

[30] N. Th. Varopoulos, Hardy-Littlewood theory for semigroups, J. Funct. Anal. 63 (1985), no. 2, 240260, DOI 10.1016/0022-1236(85)90087-4. MR803094 (87a:31011)

[31] Bruce J. West and Theo Nonnenmacher, An ant in a gurge, Phys. Lett. A 278 (2001), no. 5, 255-259, DOI 10.1016/S0375-9601(00)00781-7. MR1826567(2002a:82066)

[32] G. M. Zaslavsky, Chaos, fractional kinetics, and anomalous transport, Phys. Rep. 371 (2002), no. 6 , 461-580, DOI 10.1016/S0370-1573(02)00331-9. MR.1937584(2003i:70030) 